ARTICLE

https://doi.org/10.1038/s41467-019-11898-z

\title{
Bacterially synthesized tellurium nanostructures for broadband ultrafast nonlinear optical applications
}

\author{
Kangpeng Wang ${ }^{1,2}$, Xiaoyan Zhang ${ }^{1}$, Ivan M. Kislyakov (1) 1, Ningning Dong ${ }^{1}$, Saifeng Zhang ${ }^{1}$, Gaozhong Wang ${ }^{1,3}$, \\ Jintai Fan', Xiao Zou ${ }^{4}$, Juan $\mathrm{Du}^{4}$, Yuxin Leng ${ }^{4}$, Quanzhong Zhao ${ }^{4}$, Kan $\mathrm{Wu}^{5}$, Jianping $\mathrm{Chen}^{5}$, \\ Shaun M. Baesman6, Kang-Shyang Liao7 , Surendra Maharjan7, Hongzhou Zhang ${ }^{3}$, Long Zhang ${ }^{1,4,8}$, \\ Seamus A. Curran7, Ronald S. Oremland ${ }^{6}$, Werner J. Blau (i) ${ }^{3} \&$ Jun Wang ${ }^{1,4,8}$
}

Elementary tellurium is currently of great interest as an element with potential promise in nano-technology applications because of the recent discovery regarding its three twodimensional phases and the existence of Weyl nodes around its Femi level. Here, we report on the unique nano-photonic properties of elemental tellurium particles $[\mathrm{Te}(0)]$, as harvest from a culture of a tellurium-oxyanion respiring bacteria. The bacterially-formed nano-crystals prove effective in the photonic applications tested compared to the chemically-formed nano-materials, suggesting a unique and environmentally friendly route of synthesis. Nonlinear optical measurements of this material reveal the strong saturable absorption and nonlinear optical extinctions induced by Mie scattering over broad temporal and wavelength ranges. In both cases, Te-nanoparticles exhibit superior optical nonlinearity compared to graphene. We demonstrate that biological tellurium can be used for a variety of photonic applications which include their proof-of-concept for employment as ultrafast mode-lockers and all-optical switches.

\footnotetext{
${ }^{1}$ Laboratory of Micro-Nano Optoelectronic Materials and Devices, Laboratory of Laser and Infrared Materials, Key Laboratory of Materials for High-Power Laser, Shanghai Institute of Optics and Fine Mechanics, Chinese Academy of Sciences, Shanghai 201800, China. ${ }^{2}$ Department of Electrical Engineering, Technion-Israel Institute of Technology, Haifa 3200003, Israel. ${ }^{3}$ School of Physics, CRANN and AMBER Research Centres, Trinity College Dublin, Dublin 2 , Ireland. ${ }^{4}$ State Key Laboratory of High Field Laser Physics, CAS Center for Excellence in Ultra-intense Laser Science, Shanghai Institute of Optics and Fine Mechanics, Chinese Academy of Sciences, Shanghai 201800, China. ${ }^{5}$ State Key Laboratory of Advanced Optical Communication Systems and Networks,

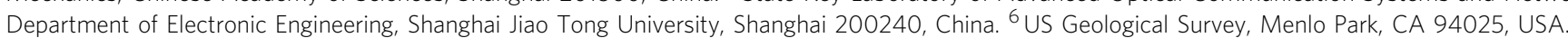
7 Institute for NanoEnergy, Department of Physics, University of Houston, Houston, TX 77204, USA. ${ }^{8}$ Center of Materials Science and Optoelectronics Engineering, University of Chinese Academy of Sciences, Beijing 100049, China. Correspondence and requests for materials should be addressed to K.W. (email: kanwu@sjtu.edu.cn) or to L.Z. (email: Izhang@siom.ac.cn) or to J.W. (email: jwang@siom.ac.cn)
} 
ow dimensional materials play increasingly powerful roles in the electronic and photonic industries ${ }^{1}$. Elemental tellurium [Te(0)] has been recently highlighted because of its unconventional properties ${ }^{2-5}$. Bulk trigonal tellurium has been predicted to have multiple Weyl nodes near its Fermi level ${ }^{5}$, opening the possibilities for high carrier-mobility and topological devices. Recently, three two-dimensional tellurene phases $(\alpha-, \beta$-, and $\gamma$ $\mathrm{Te})$ have been reported $2,3,6,7$. They exhibited excellent carriermobility which are 2-3 orders of magnitude higher than that of $\mathrm{MoS}_{2}$, the most intensively studied 2D analog ${ }^{3}$. In particular, a few layers of a-tellurium can be formed from trigonal Te via a spontaneous phase transition ${ }^{3,8}$. These properties make tellurium a promising candidate as the material of choice for nextgeneration optoelectronic and photonic devices ${ }^{2}$.

The conventional synthesis of tellurium nanomaterials relies heavily on chemical approaches that employ harsh reagents, high temperatures, and high costs associated with considerable hazardous waste disposal. The use of specific anaerobic bacteria that can process the oxyanions of Group 6 metalloids (for example, Se and $\mathrm{Te}$ ) as respiratory electron acceptors, results in their reduction to the elemental state, allowing their formation at room temperature. This approach has significant applicability in nanophotonics, as it opens the possibility of a non-polluting alternative to more commonly used chemical techniques ${ }^{9-13}$. Furthermore, the biological synthesis route was recently demonstrated for self-assembling electronic devices ${ }^{14}$. However, the physical properties of biologically formed $\mathrm{Te}(0)$-nanoparticles are largely unexplored.

In this work, we harvest elemental tellurium nanostructures formed by cultivating the anaerobic bacterium Bacillus selenitireducens with $\mathrm{Te}(\mathrm{IV})$ as its electron acceptor $9,10,15$. Nonlinear optical properties of these microbiologically synthesized tellurium (Bio-Te) nanostructures are investigated by open-aperture z-scan over broad temporal and wavelength ranges. We highlight the potential of Bio-Te nanostructures in photonic applications by building ultrafast infrared $1.5 \mu \mathrm{m}$ fiber and $2 \mu \mathrm{m}$ solid-state lasers using Bio-Te as the saturable absorber. With mode-locking and Q-switching achieved by Bio-Te, ultrafast pulse generation is observed in these lasers. In addition, an all-optical switch based on Bio-Te is demonstrated for optical fiber systems. Our results suggest that biological Te nanocrystals have the potential for a broad range of photonic applications, such as in ultrafast midinfrared lasers, and optical routing.

\section{Results}

Fabrications and characterizations of biological tellurium. The elemental tellurium nanocrystals were produced by growing Teoxyanion respiring bacteria and by harvesting the crystals after cultivation as shown in Fig. 1a (see Methods) ${ }^{10-12,16}$. The harvested Bio-Te nanostructures were aggregated into micro-pellets. However, the dense aggregations were unfavorable for the linear and nonlinear optical studies. We, therefore, employed poly $(m-$ phenylenevinylene)-co-2,5-dioctoxy-phenylenevinylene (PmPV) to disperse the $\mathrm{Te}(0)$, thereby forming a nanocomposite ${ }^{15}$ (see Supplementary Note 1). PmPV has been proven to be an ideal $\pi$ electron-rich host for many types of filler including single-walled carbon nanotubes ${ }^{17}$. The coiled conformation of PmPV enables it to wrap around the Bio-Te nanocrystals (Fig. 1b) which allows sufficiently close intermolecular proximity for $\pi-\pi$ interaction to occur. Figure 1c shows the effective dispersion of Bio-Te aggregates of Bio-Te in toluene with the addition of PmPV.

Figure 1d shows sample images obtained with a highresolution transmission electron microscope (HRTEM) and fast Fourier transformation of Bio-Te dispersed in PmPV. Lattice spacings of $0.32 \mathrm{~nm}$ and $0.59 \mathrm{~nm}$ were observed corresponding to the (101) and (001) crystal planes, respectively. This implies that Bio-Te has a triagonal crystal geometry comprised of three Teatoms per unit cell. The Te-atoms are covalently bonded, forming a series of helical chains ${ }^{18}$ that interact with each other by van der Waals forces. We also found that the applied PmPV layer surrounds the crystalline $\mathrm{Te}$, as shown in Fig. 1d. Raman spectroscopy was employed to confirm the atomic structural arrangement of Bio-Te. In Fig. 1e, the Raman spectra of both the pristine Bio-Te and the Bio-Te-PmPV composite exhibited distinct fingerprints in the crystalline trigonal $\mathrm{Te}^{19}$. The characteristic bands located at $88.6,113.6$, and $134.9 \mathrm{~cm}^{-1}$ are the first order vibration modes of bending and stretching in the Te crystal. The band observed at $261.9 \mathrm{~cm}^{-1}$ is the second-order Raman vibration. Other peaks at 219.8, 438.4, 574, 640.9, and $657.6 \mathrm{~cm}^{-1}$ belong to $\mathrm{TeO}_{2}$, which may have originated from a small amount of oxidation of $\mathrm{Te}(0)$ through exposure to ambient air over time. We also detected characteristic bands associated with PmPV in the Raman spectrum of the Bio-Te-PmPV, as indicated in Fig. 1e. Linear optical absorption of Bio-Te-PmPV composite is shown in Fig. 1f. This spectrum was achieved by subtracting the PmPV background from the spectrum of the BioTe-PmPV composite. A typical broad absorption band centered at $1.9 \mathrm{eV}(\sim 650 \mathrm{~nm})$ is observed, which is caused by the transitions from the $p$-nonbonding valence band triplet to the $p$-antibonding conduction band triplet ${ }^{20,21}$.

The steady-state photoluminescence (PL) spectra of the BioTe-PmPV and PmPV are shown on the right-hand side of Fig. 1f. The PL envelopes of both samples were quite similar, showing intrinsic PLs with a broad peak between $450 \mathrm{~nm}$ and $650 \mathrm{~nm}$. This similarity indicates that PmPV molecules retained their PL, whereas the Te crystal did not exhibit PL. However, the PL intensity of the Bio-Te-PmPV was approximately one order of magnitude lower than that of the pure PmPV under the same excitation, suggesting that the quench of the luminescence of PmPV was caused by the Bio-Te nanocrystal. To gain a comprehensive understanding of this effect, we carried out PL decay kinetics measurements of both samples. As shown in Fig. 1g, the PL decay of Bio-Te-PmPV was faster than that of pure PmPV for the first few ns, as marked by the blue shaded region (see inset). We employed a tri-exponential model to fit the PL decay curves, and the results are shown in Supplementary Table 1. The two dominant PL decay lifetimes were fitted to be $\tau_{1} \sim 338$ ps and $\tau_{2} \sim 1.09 \mathrm{~ns}$ for Bio-Te-PmPV, while $\tau_{1} \sim 460 \mathrm{ps}$ and $\tau_{2}$ $\sim 1.11 \mathrm{~ns}$ were observed for PmPV, respectively. The faster PL decay in Bio-Te-PmPV implies a quenching effect caused by by the Bio-Te nanocrystals. A possible explanation for this quenching is the existence of energy transfer from the excited donor PmPV molecules to the acceptor Bio-Te nanocrystals which is evidenced by the overlap of the PmPV PL peak and the Bio-Te absorption band (red shaded area in Fig. 1f). That is, the Te-acceptor nanocrystals consumed the energy of excited carriers and relaxed them to the ground state non-radiatively, leading to the drop of the PL intensity in the first few ns (1-10 ns).

Nonlinear optical properties of biological tellurium. To reveal the nonlinear absorptive responses of Bio-Te-PmPV, we carried out a series of open-aperture $\mathrm{z}$-scans with different wavelength excitations using fs pulses (Fig. 2). With the excitation set at $800 \mathrm{~nm}$ and applying $200 \mathrm{~nJ}$ pulses (maximum of $175 \mathrm{GW} \cdot \mathrm{cm}^{-2}$ ), the Bio-Te-PmPV exhibited saturable absorption (SA), that is, the optical transmission increases with the incident beam intensity when the sample position $z$ approaches the focal point of the focusing lens $(z=0 \mathrm{~mm})$ (Fig. 2a). However, the SA response was not observed in PmPV solutions (the squares in Fig. 2a), indicating that the SA response was caused solely by the Te 
a
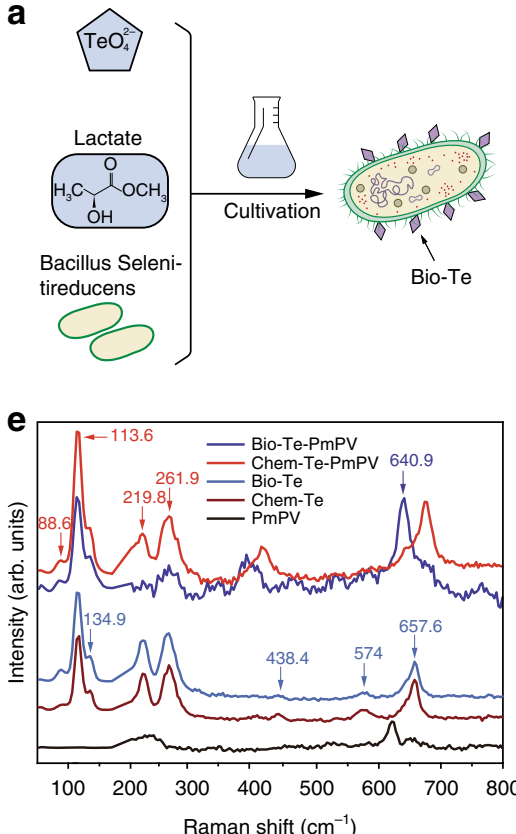
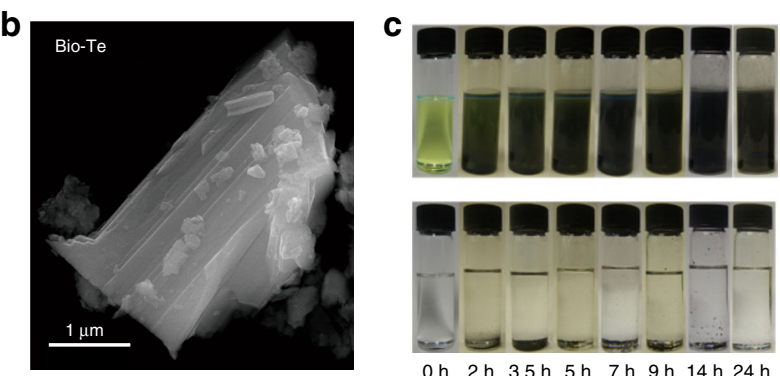

$0 \mathrm{~h} \quad 2 \mathrm{~h} 3.5 \mathrm{~h} 5 \mathrm{~h} \quad 7 \mathrm{~h} 9 \mathrm{~h} 14 \mathrm{~h} 24 \mathrm{~h}$
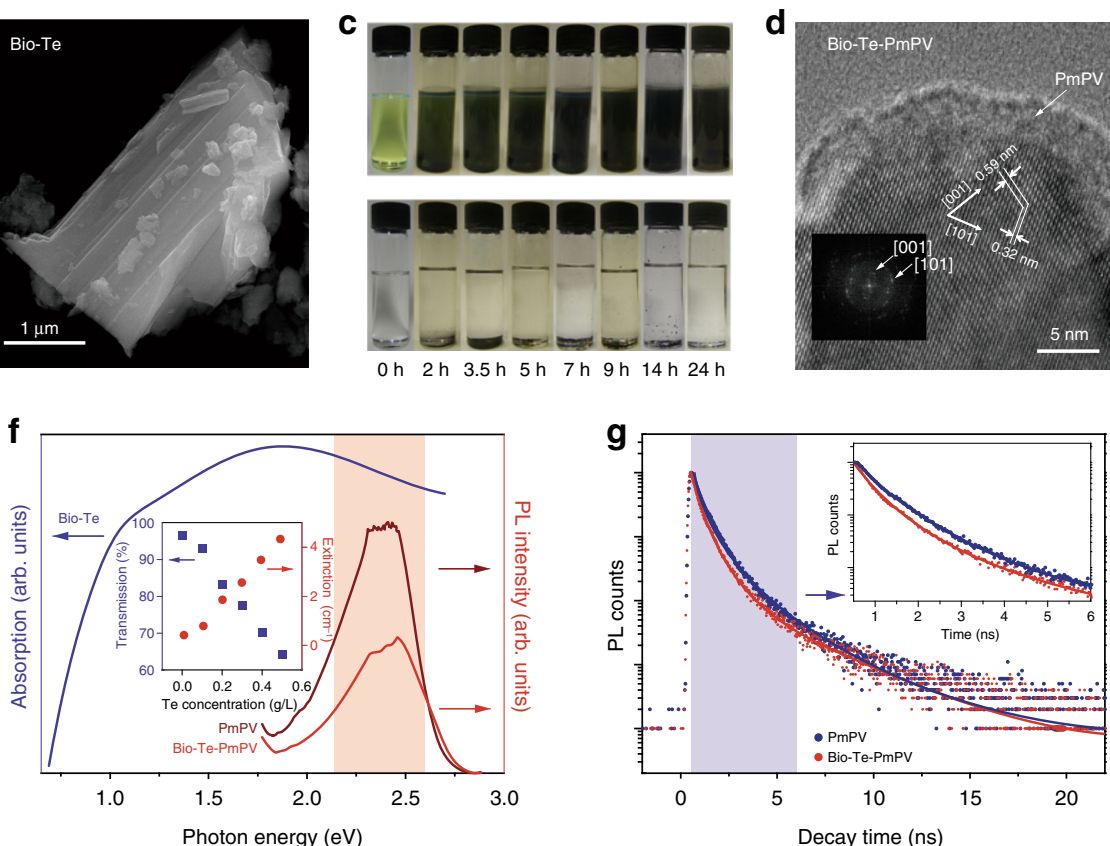

Fig. 1 Synthesis and characterization of biological synthesized tellurium (Bio-Te). a Synthesis scheme of tellurium nanocrystals by anaerobic bacteria, Bacillus selenitireducens. b Image of Bio-Te crystalline nano-flake taken by scanning transmission electron microscope. c Dispersion of Bio-Te in PmPV/ toluene (upper) and in toluene only (bottom) with increasing stirring times on the X-axis, showing the preparation of Bio-Te-PmPV composites. PmPV is abbreviation of poly ( $m$-phenylenevinylene)-co-2,5-dioctoxy-phenylenevinylene. $\mathbf{d}$ Transmission electron microscopy image of a Bio-Te crystalline nanoflake wrapped by PmPV layers. Inset is the image after fast Fourier transformation (FFT). e Raman spectra of Bio-Te, Bio-Te-PmPV, chemically synthesized tellurium nanocrystals (Chem-Te), Chem-Te-PmPV, and PmPV. $\mathbf{f}$ Left: the absorption spectrum of Bio-Te. This curve was obtained by subtracting the absorption of PmPV (0.5 g/L in toluene) from that of Bio-Te-PmPV. Right: photoluminescence (PL) spectra of Bio-Te-PmPV and pure PmPV. Inset: optical linear transmission and extinction coefficient as functions of Te concentrations at $532 \mathrm{~nm}$. $\mathbf{g}$ The PL decay kinetics of PmPV and Bio-Te-PmPV at $528 \mathrm{~nm}$ excitation. The inset shows a blow up of the blue shaded region, showing the quenching effect caused by tellurium
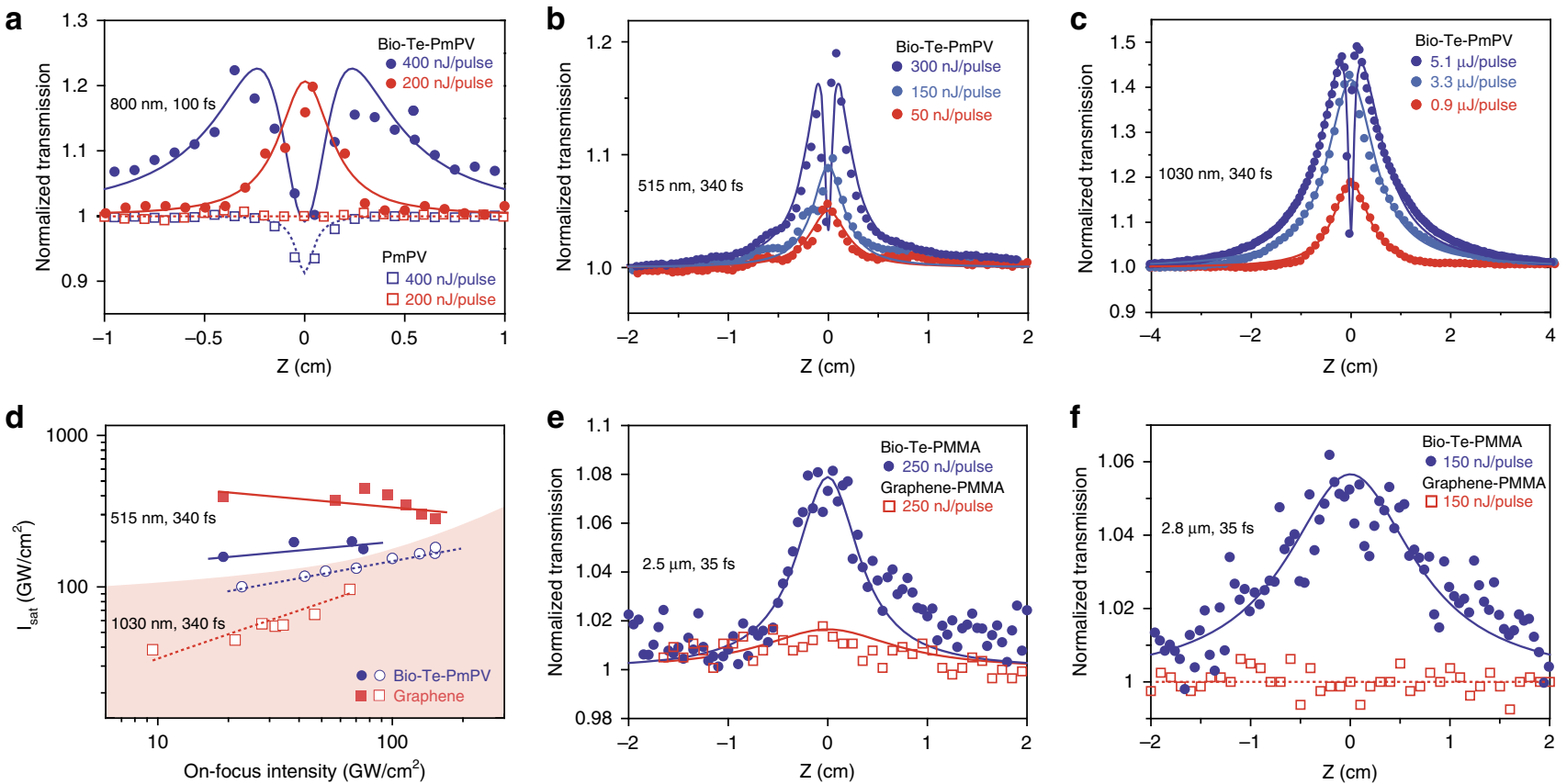

Fig. 2 Open-aperture z-scan results of the biological synthesized tellurium samples. a Experimental results with fs pulses, $800 \mathrm{~nm}$. Bio-Te-PmPV (solid circles); PmPV (hollow squares). Solid lines: fitted z-scan curves using equation (1); Dashed lines are for visual guide. b, c Experimental results with 340 fs laser at $515 \mathrm{~nm}(\mathbf{b})$ and $1030 \mathrm{~nm}$ (c). d Saturated intensity $I_{\text {sat }}$ of Bio-Te-PmPV and graphene dispersion as functions of the laser intensity at $1030 \mathrm{~nm}$ and $515 \mathrm{~nm}$. e, $\mathbf{f}$ Mid-infrared open z-scans of Bio-Te and graphene polymethyl methacrylate (PMMA) films at $2.5 \mu \mathrm{m}$ and $2.8 \mu \mathrm{m}$ wavelengths, showing better saturable absorptive responses of Bio-Te than those of graphene 
nanocrystals. Because the excitation photon energy $(1.55 \mathrm{eV})$, is much larger than the Te bandgap ${ }^{5}(0.323 \mathrm{eV})$, the conduction band of Te can be filled at high photon intensities. This bandfilling leads to Pauli blocking and thereafter saturable absorption. As the excitation pulse energy increased to $400 \mathrm{~nJ}$ (maximum of $350 \mathrm{GW} \cdot \mathrm{cm}^{-2}$ ), a dip appeared around the center of the $\mathrm{z}$-scan traces, implying that a combined effect of SA and nonlinear extinction (NLE) was taking place. This NLE effect may be attributed to the multiphoton absorption brought by $\mathrm{PmPV}^{15}$. The nonlinear optical (NLO) coefficients of PmPV and Bio-Te can be extracted from $\mathrm{z}$-scan traces using the following photon transport equation:

$$
\frac{d I}{d z}=-\frac{\alpha_{0} I}{1+\frac{I}{I_{\mathrm{sat}}}}-\beta I^{2}
$$

where $\alpha_{0}$ is the linear absorption coefficient, $I$ is the photon intensity, $z$ is the distance traveled by light in the NLO medium, $I_{\text {sat }}$ is the saturated intensity contributed by Bio-Te, and $\beta$ is the NLE coefficient contributed by PmPV. For laser excitations at $515 \mathrm{~nm}$ and $800 \mathrm{~nm}$ wavelengths, PmPV exhibited a two-photon absorption, therefore $\beta$ can be regarded as the two-photon absorption coefficient $\left(\beta_{2 \mathrm{PA}}\right)$ of PmPV. For laser excitation at $1030 \mathrm{~nm}, \mathrm{PmPV}$ exhibited a three-photon absorption profile, therefore $\beta=\gamma_{3 \mathrm{PA}} I$, where $\gamma_{3 \mathrm{PA}}$ is the three-photon absorption coefficient of PmPV. The imaginary part of the third-order NLO susceptibility, $\operatorname{Im} \chi^{(3)}$, which is directly related to NLO coefficient $\alpha_{\mathrm{NL}}$, is calculated from $\operatorname{Im} \chi^{(3)}=\left[10^{-7} c \lambda n^{2} /\left(96 \pi^{2}\right)\right] \alpha_{\mathrm{NL}}$, where $c$ is the speed of light, $\lambda$ is the excitation wavelength, and $n$ is the refractive index. In the case of Bio-Te, $\alpha_{\mathrm{NL}}$ was fully attributed to saturable absorption, that is, $\alpha_{\mathrm{NL}} \cong-\alpha_{0} / I_{\text {sat }}$ (Fig. 2). The figure of merit (FOM) of Bio-Te for the third-order optical nonlinearity is defined as $\mathrm{FOM}=\left|\operatorname{Im} \chi^{(3)} / \alpha_{0}\right|$. The fitted $\mathrm{z}$-scan curves using Eq. (1) are presented in Fig. 2a, and correspond well correspondence with the experimental data obtained for the $200 \mathrm{~nJ} /$ pulse to $600 \mathrm{~nJ} /$ pulse excitations. The average saturable intensity $I_{\text {sat }}$ of Bio-Te at $800 \mathrm{~nm}$ was determined to be $261 \pm 176 \mathrm{GW} \cdot \mathrm{cm}^{-2}$. This implies that the SA of Bio-Te was at least comparable to that of black phosphorus (BP) at $800 \mathrm{~nm}$, which was reported as $335 \pm 43 \mathrm{GW} \cdot \mathrm{cm}^{-2} 22$.

Figure $2 \mathrm{~b}, \mathrm{c}$ show the $\mathrm{z}$-scan traces of Bio-Te-PmPV for $340 \mathrm{fs}$ at $515 \mathrm{~nm}$ and $1030 \mathrm{~nm}$ laser pulses, which indicate the broadband saturable absorption by Te-nanoparticles over the range of visible to near-infrared (NIR) wavelengths. The linear absorption coefficients $\left(\alpha_{0}\right)$ of Bio-Te-PmPV at 1030 and $515 \mathrm{~nm}$ were $6.47 \mathrm{~cm}^{-1}$ and $6.17 \mathrm{~cm}^{-1}$, respectively. For higher intensity laser excitations at 800,1030 , and $515 \mathrm{~nm}$, the multi-photon absorption in PmPV resulted in an obvious turning point in optical transmission around $z=0 \mathrm{~cm}$ for Bio-Te-PmPV. These experimental data were fitted using Eq. (1) (Fig. 2b, c). As noted previously at $800 \mathrm{~nm}$, the PmPV only contributed to multiphoton absorption while SA was caused by the Bio-Te. Figure $2 \mathrm{~d}$ summarizes the saturation intensity $\left(I_{\text {sat }}\right)$ as a function of the onfocus beam intensity for a series of $z$-scans. In Bio-Te, $I_{\text {sat }}$ increased slightly with laser intensity with a median value of $145 \pm 23 \mathrm{GW} \cdot \mathrm{cm}^{-2}$ for $1030 \mathrm{~nm}$, and $201 \pm 35 \mathrm{GW} \cdot \mathrm{cm}^{-2}$ for $515 \mathrm{~nm}$. The median $\operatorname{Im} \chi^{(3)}$ and FOM of Bio-Te were respectively determined to be $-(2.76 \pm 0.58) \times 10^{-14}$ esu and $(4.27 \pm 0.91) \times$ $10^{-15} \mathrm{esu} \cdot \mathrm{cm}$ for $1030 \mathrm{~nm}$, and $-(1.07 \pm 0.11) \times 10^{-14} \mathrm{esu}$ and $(1.74 \pm 0.18) \times 10^{-15} \mathrm{esu} \cdot \mathrm{cm}$ for $515 \mathrm{~nm}$. The $I_{\text {sat }}$ for Bio-Te at $1030 \mathrm{~nm}$ was approximately one-third that of BP, implying a much stronger SA response to infrared excitation ${ }^{23}$.

We further demonstrated the saturable absorptive response of Bio-Te at mid-infrared wavelengths with $\sim 35 \mathrm{fs}$ laser pulses. Because of the strong two-photon absorption of the PmPV-based films in the mid-infrared (MIR) wavelengths, we used polymethyl methacrylate (PMMA) to host the Bio-Te nanocrystals for our MIR measurements (see Methods). Figure 2e, f present the z-scan results of Bio-Te-PMMA at $2.5 \mu \mathrm{m}$ and $2.8 \mu \mathrm{m}$ wavelengths, respectively. The noise level is higher than that in NIR because of the relatively low signal-to-noise ratio of the detector used (a PbS photodiode). While the host PMMA exhibited negligible NLO responses (see Supplementary Fig. 1a), the composite Bio-TePMMA film showed obvious saturable absorption at both $2.5 \mu \mathrm{m}$ and $2.8 \mu \mathrm{m}$. This difference implies that the Bio-Te is the only material that contributed to the saturable absorption in Fig. 2e, f. The saturated intensities of Bio-Te-PMMA were fitted as 220 $\mathrm{GW} \cdot \mathrm{cm}^{-2}$ and $245 \mathrm{GW} \cdot \mathrm{cm}^{-2}$ for $2.5 \mu \mathrm{m}$ and $2.8 \mu \mathrm{m}$, respectively. The field of ultrafast mid-infrared fiber lasers is important and active, and these saturable absorptive responses imply that the Bio-Te can serve as a passive mode-locker for such lasers, especially those based on erbium and holmium ions.

We compared the SA responses of Bio-Te with graphene, a well-known NLO material for broadband SA ${ }^{24-26}$, over the range of the visible to mid-infrared (MIR) spectrum. In the visible to near-infrared range, we used graphene dispersed in N-Methyl-2pyrrolidone (graphene-NMP, see Supplementary Note 1) for comparative studies. Compared to the SA response of graphene, the Bio-Te-PmPV response was stronger at $515 \mathrm{~nm}$, comparable at $800 \mathrm{~nm}$, but inferior at $1030 \mathrm{~nm}$ (see Supplementary Fig. 2). In the MIR range, we compared tellurium with graphene in Fig. 2e, $\mathrm{f}$ with PMMA as the host material (see Methods and Supplementary Note 1), where the Bio-Te exhibited much stronger SA than graphene at both $2.5 \mu \mathrm{m}$ and $2.8 \mu \mathrm{m}$ wavelengths. This weak NLO response of graphene may be partially attributed to its twophoton absorption ${ }^{27}$, which is more obvious at higher laser irradiance (see the dip of transmission around $z=0 \mathrm{~cm}$ in

\begin{tabular}{|c|c|c|c|c|c|c|}
\hline Laser & Sample & $T(\%)$ & $\alpha_{0}\left(\mathrm{~cm}^{-1}\right)$ & $I_{\text {sat }}\left(G W \cdot \mathrm{cm}^{-2}\right)$ & $\operatorname{Im} \chi^{(3)}\left(\times 10^{-14} \mathrm{esu}\right)$ & FOM $\left(\times 10^{-15}\right.$ esu.cm $)$ \\
\hline \multirow[t]{2}{*}{$515 \mathrm{~nm}, 340 \mathrm{fs}$} & Bio-Te-PmPV & 54.0 & 6.17 & $201 \pm 35$ & $-1.07 \pm 0.11$ & $1.74 \pm 0.18$ \\
\hline & Graphene-NMP & 46.1 & 7.74 & $364 \pm 57$ & $-0.67 \pm 0.09$ & $0.86 \pm 0.11$ \\
\hline \multirow[t]{2}{*}{$800 \mathrm{~nm}, 100 \mathrm{fs}$} & Bio-Te-PmPV & 45.0 & 7.99 & $261 \pm 176$ & $-1.2 \pm 0.4$ & $1.47 \pm 0.52$ \\
\hline & Graphene-NMP & 16.5 & 18.0 & 910 & -0.968 & 0.54 \\
\hline \multirow[t]{2}{*}{$1030 \mathrm{~nm}, 340 \mathrm{fs}$} & Bio-Te-PmPV & 52.4 & 6.47 & $145 \pm 23$ & $-2.76 \pm 0.58$ & $4.27 \pm 0.91$ \\
\hline & Graphene-NMP & 52.9 & 6.37 & $56 \pm 11$ & $-5.9 \pm 2.1$ & $9.3 \pm 3.2$ \\
\hline \multirow[t]{2}{*}{$2500 \mathrm{~nm}, 35 \mathrm{fs}$} & Bio-Te-PMMA & 74.2 & 25.7 & 220 & -20.5 & 7.99 \\
\hline & Graphene-PMMA & 75.7 & 78.9 & 900 & -15.4 & 1.95 \\
\hline \multirow[t]{2}{*}{$2800 \mathrm{~nm}, 35 \mathrm{fs}$} & Bio-Te-PMMA & 29.6 & 105 & 245 & -84.1 & 8.03 \\
\hline & Graphene-PMMA & 71.2 & 96.2 & - & - & - \\
\hline
\end{tabular}

Saturable absorption coefficients, $I_{\mathrm{sat}}$ of Bio-Te and graphene obtained from open-aperture $z$-scan experiments under various femtosecond laser irradiations, noting that the graphene does not show saturable absorption at $2.8 \mu \mathrm{m}$ 

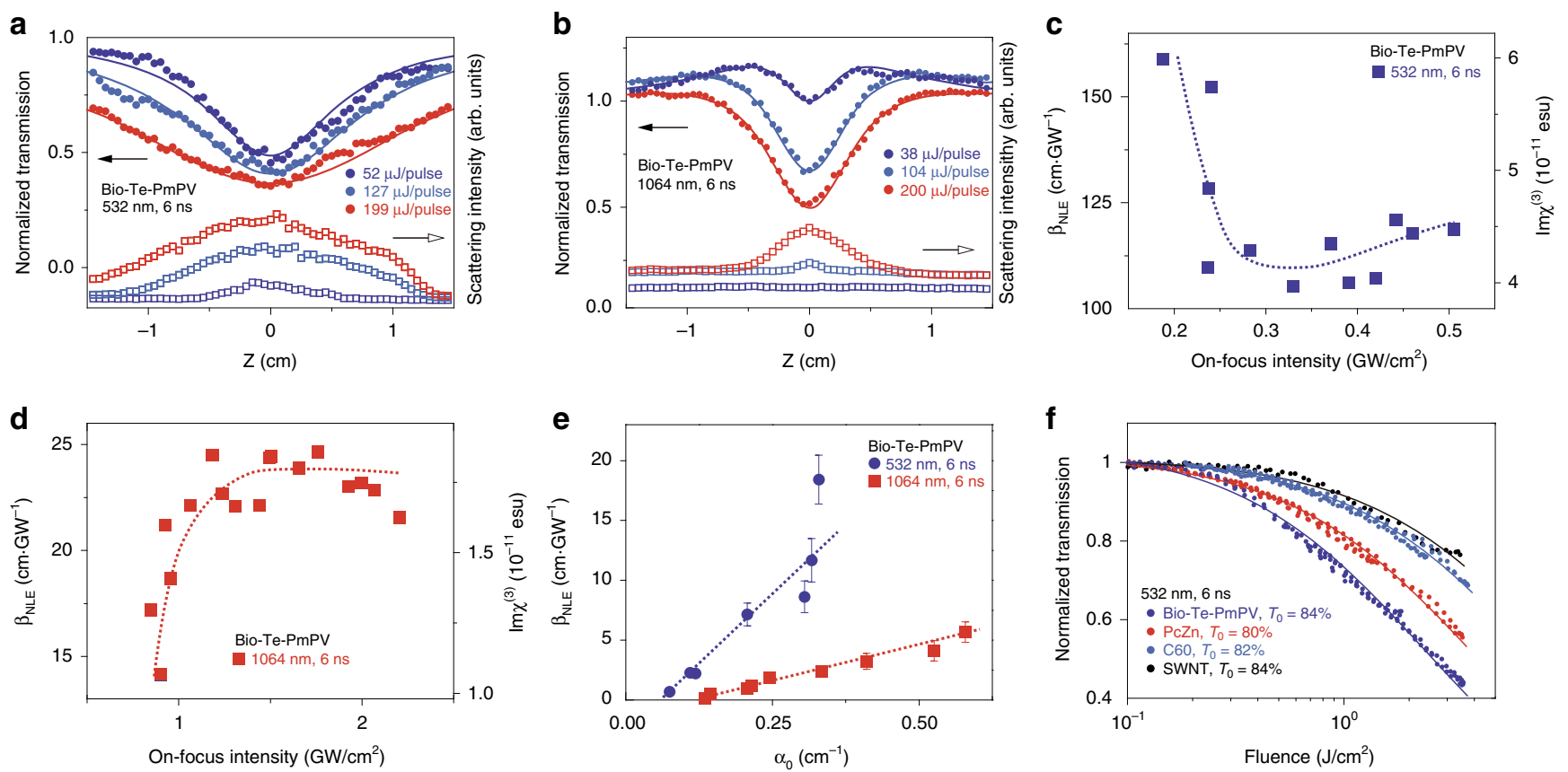

Fig. 3 Nonlinear optical responses to ns pulses. a, b Circles: normalized transmission of Bio-Te-PmPV as a function of $z$ at $532 \mathrm{~nm}$ and $1064 \mathrm{~nm}$; Squares: light intensity scattered by the sample at 35 degrees to the laser's direction; Lines: $z$-scan fitting results. c, $\mathbf{d}$ Effective nonlinear extinction (NLE) coefficient $\beta_{\mathrm{NLE}}$ and corresponding $\operatorname{Im} \chi^{(3)}$ as a function of on-focus intensity for ns pulses at 532/1064 nm. e Effective nonlinear extinction coefficient $\beta_{\mathrm{NLE}}$ as a function of linear absorption coefficient $\alpha_{0}$ with error bars indicating s.e.m. $\mathbf{f}$ Comparison of optical limiting performance of Bio-Te-PmPV, PcZn ( $t$ $\mathrm{Bu}_{4} \mathrm{PcZn}$ ), $\mathrm{C}_{60}$, and single-walled carbon nanotube (SWNT) dispersions at $532 \mathrm{~nm}, 6 \mathrm{~ns}$ irradiation

Supplementary Fig. 1b). The NLO coefficients of graphene and Bio-Te are presented in Table 1. The strong SA for fs pulses in the infrared region signifies that the biologically synthesized Te holds great technical potential as a passive mode-locker for generating ultra-short laser pulses, which is discussed further later in this paper.

To study the NLO response of Bio-Te-PmPV to nanosecond pulses, we performed z-scans using $6 \mathrm{~ns}$ pulses from a $10 \mathrm{~Hz} \mathrm{Q}$ switched Nd:YAG laser. The scattered light was collected using a convex lens at $\sim 35^{\circ}$ to the direction of the incident beam. The results are shown in Fig. 3a, b. Bio-Te-PmPV exhibited NLE for z-scan traces from $52 \mu \mathrm{J}$ to $199 \mu \mathrm{J}$ pulse energy at $532 \mathrm{~nm}$ (Fig. 3e). Because PmPV only exhibited multi-photon absorption at $532 \mathrm{~nm}$ and $1064 \mathrm{~nm}$, no contribution of scattering light would be expected ${ }^{15}$; therefore, the strong scattering signal was attributed to $\mathrm{Bio}-\mathrm{Te}^{28}$. The absorption of laser pulse energy by $\mathrm{Te}$ nanostructures induced micro-bubbles and/or plasmas in the medium, causing strong Mie scattering and hence the attenuation of incident light. The SA effect was minimal in Bio-Te-PmPV (Fig. 3a), which may have been overwhelmed by the much stronger NLE effect. Similar to the fs measurements, we can neglect the effect of PmPV because of its small absorption $(0.346$ $\mathrm{cm}^{-1}$ at $532 \mathrm{~nm}$, with a total absorption coefficient of $3.47 \mathrm{~cm}^{-1}$ ). In this case, the photon transport equation can be simplified as:

$$
\frac{d I}{d z}=-\left(\alpha_{0}+\beta_{\mathrm{NLE}} I\right) I
$$

where $\beta_{\mathrm{NLE}}$ is the effective NLE coefficient. The fitted z-scan traces according to Eq. (2) corresponded well with the experimental results as shown in Fig. 3a. For $1064 \mathrm{~nm}$ (Fig. 3b), the combined effects of SA and NLE were observed in Bio-TePmPV. SA was the dominant effect in the low-intensity region, while NLE dominated in the high-intensity regime. In this case, we still employed Eq. (1) for z-scan fitting, and the results are shown in Fig. 3b. Symmetric valleys around $z=0$ were observed giving rise to NLE effects; however, $\beta_{\mathrm{NLE}}$ is now attributed to prominent nonlinear scattering. The plot of $\beta_{\mathrm{NLE}}$ as a function of on-focus intensities for $532 \mathrm{~nm}$ and $1064 \mathrm{~nm}$ pulses are given in Fig. 3c, d, which may reflect high-order nonlinearities that accompany with the nonlinear scattering in the Te composites. To investigate the relationship between $\beta_{\mathrm{NLE}}$ and linear absorptive coefficient $\alpha_{0}$, we conducted a series of z-scans with different concentrations of Bio-Te dispersions (Fig. 3e). The $\beta_{\mathrm{NLE}}$ showed a linear dependence of $\beta_{\mathrm{NLE}}=47.3\left(\alpha_{0}-0.06\right)$ $\left(\mathrm{cm} \cdot \mathrm{GW}^{-1}\right)$ for $532 \mathrm{~nm}$, and $\beta_{\mathrm{NLE}}=12\left(\alpha_{0}-0.014\right)\left(\mathrm{cm} \cdot \mathrm{GW}^{-1}\right)$ for $1064 \mathrm{~nm}$.

The broadband NLE exhibited by Bio-Te-PmPV (Fig. 3) can be exploited for laser protective applications ${ }^{28-30}$. To evaluate this potential, systematic comparisons with several well-known NLE materials were carried out (Fig. 3f) which included PcZn $\left(t-\mathrm{Bu}_{4} \mathrm{PcZn}\right), \mathrm{C}_{60}$ fullerene, and single-walled carbon nanotubes (SWNTs). Additional results comparing Bio-Te-PmPV with graphene and graphene oxide (GO) dispersions ${ }^{28-32}$ are shown in Supplementary Fig. 3. Our results demonstrate that Bio-TePmPV possesses a much superior optical limiting capability compared to the rest of the other materials under the consideration including graphene and GO, which are considered to be highly promising materials for laser protection. The strong optical limiting capability of Bio-Te-PmPV may benefit from two useful properties of Bio-Te nanoparticles: fast local temperature rises from the large ratio of absorption cross-section $\sigma$ to heat capacity $C_{\mathrm{p}}$, and its low phase-transition melting temperature at $450{ }^{\circ} \mathrm{C}$ and boiling temperature at $988^{\circ} \mathrm{C}\left(\sigma / C_{\mathrm{p}}\right.$ of Te is $\sim 4.9$ times larger than that of carbon materials such as graphite, and graphite only exhibits sublimation at $\sim 4000^{\circ} \mathrm{C}$. See Supplementary Note 2 for more details). With these properties, the Te nanocrystals can contribute many micro-inhomogeneities (droplets and bubbles) in suspension, thus resulting in strong nonlinear scattering. 
a
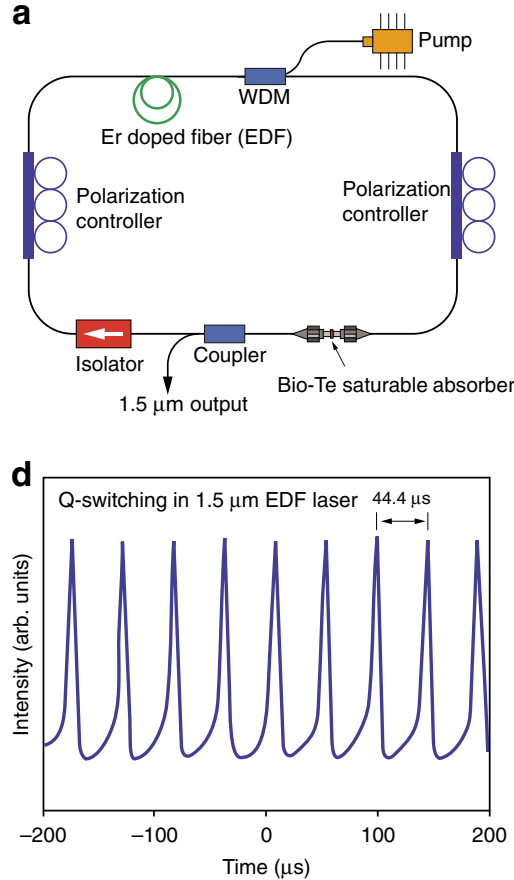

b

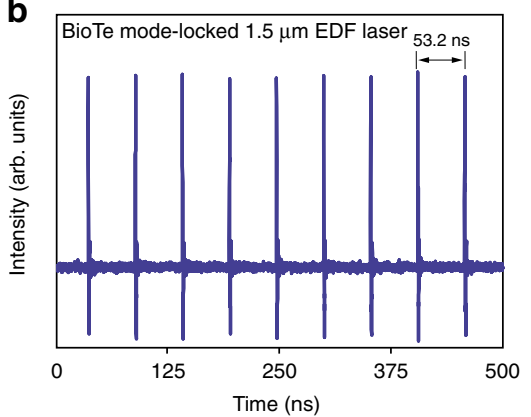

e

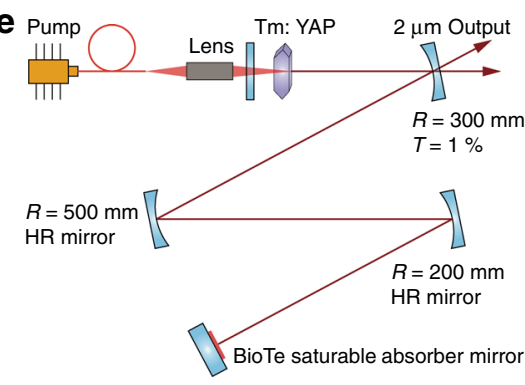

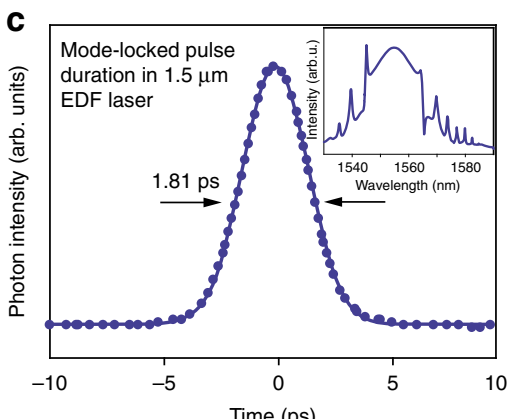

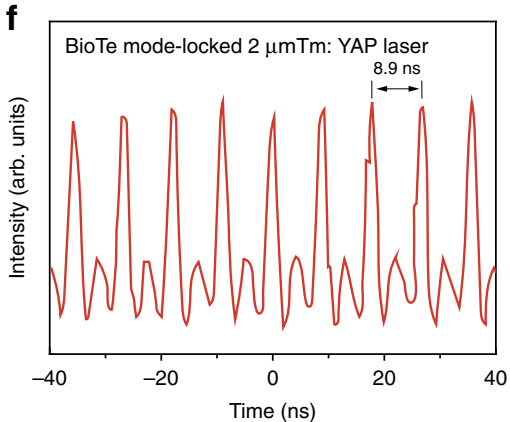

Fig. 4 Ultrafast laser pulse generation using biologically synthesized tellurium. a Scheme of the erbium-doped fiber laser with a Bio-Te-saturable absorber. b Mode-locked pulse train generated by the erbium fiber laser with repetition rate of $18.8 \mathrm{MHz}$. c Auto-correlation measurement of Bio-Te mode-locked laser pulses. The sech ${ }^{2}$ fitting indicates a 1.81 ps pulse width. The inset shows the spectrum of the mode-locked pulses. $\mathbf{d}$ Q-switched pulse train generated in the EDF fiber laser. e Diagram of the Tm: YAP $2 \mu \mathrm{m}$ laser with Bio-Te saturable absorber mirror acting as a passive optical modulator. $\mathbf{f}$ Mode-locked pulses generated in the same Tm: YAP laser with the a $\sim 112.3 \mathrm{MHz}$ repetition rate and $1.6 \mathrm{~ns}$ pulse duration

A comparison of NLO responses between biologically and commercially available (Sigma Aldrich) chemically synthesized tellurium nanocrystals (Chem-Te) was also carried out. We followed the same procedure to prepare Chem-Te-PmPV composites. As shown in Fig. 1c, Chem-Te and Chem-TePmPV have similar Raman fingerprints as their Bio-Te counterparts. The comparison of nonlinear optical performances of Chem-Te and Bio-Te are presented in Supplementary Figs. 2d and $3 \mathrm{~b}$. We observed a strong SA in the NIR spectrum in the fs regime and a broadband optical limiting for ns pulses in ChemTe-PmPV, which were comparable to Bio-Te. These results demonstrate that the microbiologically synthesized Te material has equivalent photonic properties compared to commercially available Te.

Demonstrations of biological tellurium for photonic applications. We examined the potential of Bio-Te as a material for photonic uses and demonstrated that Bio-Te nanocrystals can be used for passive mode-locking and Q-switching in infrared lasers near $1.55 \mu \mathrm{m}$ (Fig. $4 \mathrm{a}-\mathrm{d}$ ). The development of ultra-fast fiber lasers has attracted much attention because of their high stability, large gain, and robust mode confinements ${ }^{26,33}$. To build a Bio-Te ultrafast fiber laser we integrated the Bio-Te nanocrystals into $\mathrm{PMMA}^{34}$ (see Methods). Bio-Te film was coated on the face of a fiber connector and inserted into the cavity of an erbium-doped fiber (EDF) laser. With the help of the Bio-Te saturable absorber, we observed self-starting modelocking with $360 \mathrm{~mW}$ pump power (Fig. 4b). A repetition rate of $18.8 \mathrm{MHz}$ was achieved corresponding to a cavity length of $\sim 10.6 \mathrm{~m}$. The output spectrum of the Bio-Te EDF laser is shown in the inset of Fig. $4 \mathrm{c}$, where few narrow sidebands are distributed along both sides of the $1.55 \mu \mathrm{m}$ peak. The formation of these Kelly spectral sidebands was due to the periodic perturbation in the laser cavity, indicating that the laser was operated in the soliton region. Figure $4 \mathrm{c}$ also shows the autocorrelation trace of the Bio-Te EDF laser. The pulsed duration was fitted to $1.81 \mathrm{ps}$ with the $\mathrm{sech}^{2}$ function, compared to the 1.46 ps pulse duration obtained for black phosphorus ${ }^{35}$. The maximum power output of ultra-fast pulses was $1.95 \mathrm{~mW}$ (see Supplementary Fig. 4), corresponding to an output pulse energy of $103.7 \mathrm{pJ}$. In addition to our observations on mode-locking, we followed the Q-switching properties of the Bio-Te saturable absorber, as shown in Supplementary Fig. 5. Q-switching was self-started with $\sim 40 \mathrm{~mW}$ pump power and disappeared at $>110 \mathrm{~mW}$ pump power. As the pump power increased from $0 \mathrm{~mW}$ to $110 \mathrm{~mW}$, the laser repetition rate increased from $7.5 \mathrm{kHz}$ to $30 \mathrm{kHz}$, and the pulse width varied from $7 \mu$ s to $12 \mu \mathrm{s}$ (Supplementary Fig. 5). Compared to the $10.5 \mu$ s pulse duration in a black phosphorus Q-switching EDF laser with $>160 \mathrm{~mW}$ pumping ${ }^{35}$, our Bio-Te laser could achieve a $7 \mu$ s pulse duration with merely $90 \mathrm{~mW}$ pumping power, which is a significant reduction in power needs. This comparison implies that Bio-Te is a more promising material than black phosphorus as a Q-switcher. The maximum Q-switched pulse energy of this fiber laser was found to be $10.3 \mathrm{~nJ}$ with $100 \mathrm{~mW}$ pump power (see Supplementary Fig. 5a, c). The pulse train is shown in Fig. 4d.

Tellurium has a small bandgap of $0.335 \mathrm{eV}$, comparable to that of black phosphorus ${ }^{5,22}$. This suggests that Bio-Te can be used to generate a mid-infrared ultrafast laser pulse (up to $3.7 \mu \mathrm{m}$ wavelength $)^{35,36}$. We demonstrated this potential in a Tm:YAP $2 \mu \mathrm{m}$ solid laser (Fig. 4e). A Bio-Te-PMMA film was coated onto a highly reflective dielectric mirror, thus acting as a saturable absorptive mirror (SAM). We achieved mode-locking using BioTe-SAM with a $112.3 \mathrm{MHz}$ repetition rate and a $1.6 \mathrm{~ns}$ pulse width (Fig. 4f). Moreover, the Q-switching operation was also demonstrated with maximum pulse energy of $58 \mu \mathrm{J}$ and a 
a
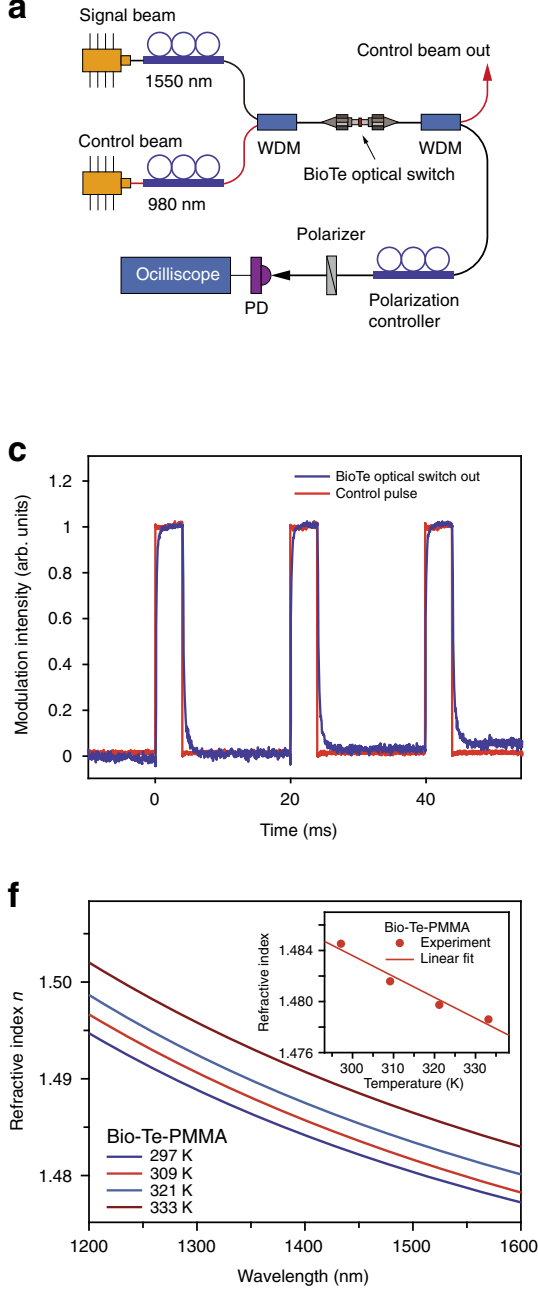

b
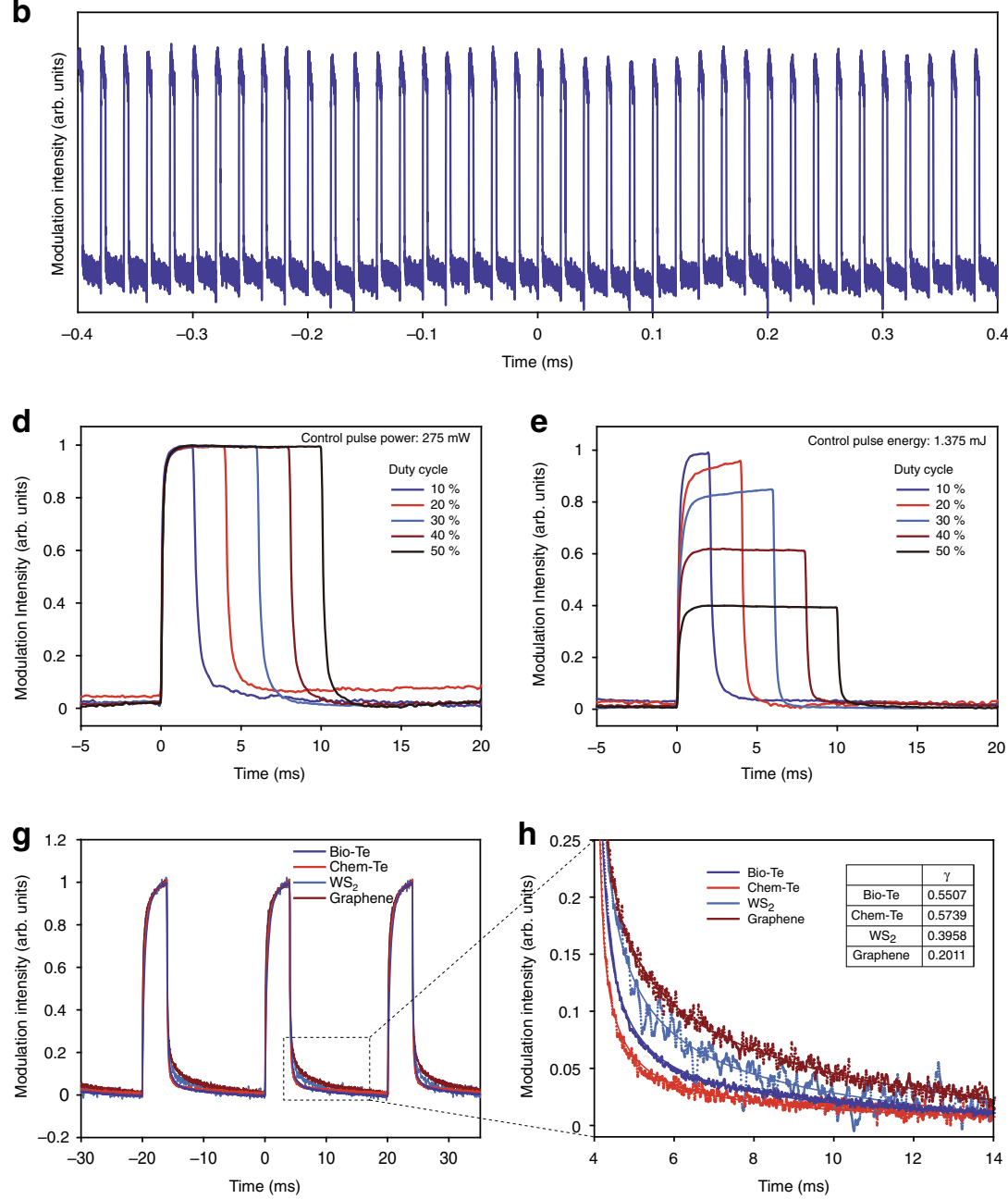

Fig. 5 Demonstration of all-optical switch based on biologically synthesized tellurium. a Scheme for testing the performance of a Bio-Te optical-switch based on polarization interference. $\mathbf{b}$ Long-term output waveform of the Bio-Te optical-switch, indicating its output stability. The average power and duty cycle of the controlling $980 \mathrm{~nm}$ pulses were $55 \mathrm{~mW}$ and 20\%, respectively. c Comparison of the signal input to the output from the Bio-Te optical switch. The rise time and fall time were measured as $276.3 \mu \mathrm{s}$ and $563.0 \mu \mathrm{s}$. d, e Effect of duty cycles of the control pulse on the output of the Bio-Te optical switch when the control pulse's peak intensity (d) or pulse energy (e) remains constant. f Refractive index of Bio-Te-PMMA film measured at different temperatures by an ellipsometer. Inset: the refractive index at $1.55 \mu \mathrm{m}$ as a function of temperature. $\mathbf{g}$ Comparison of the output waveforms based on various films serving as working material for optical switching, showing the recovery lifetimes of different materials. $\mathbf{h}$ Enlarged image from the marked area in $(\mathrm{g})$ showing the performance in signal decay of different materials once the control pulse was turned off. The falling times are determined to be $764 \mu \mathrm{s}$ and $476 \mu$ for Bio-Te and Chem-Te, and are $1.440 \mathrm{~ms}$ and 2.200 ms for $\mathrm{WS}_{2}$ nanoflakes and graphene, respectively. Solid lines are fitted to the power law, $y \propto t^{-\gamma}$. Inset table: the exponent, $\gamma$, from the fit of the power law

minimum pulse duration of $11 \mu$ s (see Supplementary Fig. 6). The maximum power of the Bio-Te Q-switching laser reached $400 \mathrm{~mW}$, suggesting that Bio-Te has a high thermal stability. The versatility of the Bio-Te SA observed from the experimental results suggests that it could be a promising photonic material for mid-infrared lasers.

We investigated the potential application of biological tellurium as an optical switching device in a fiber optic system. All-optical switching is of great importance for signal processing in many applications including optical communication and computation $^{37,38}$. Our Bio-Te optical switch was based on the polarization-dependent thermo-optic effect of Te-PMMA thin film (See Supplementary Note 3). Figure 5a shows the scheme for a Bio-Te optical switch using polarization interference ${ }^{37}$. The $1.55 \mu \mathrm{m}$ signal beam and $980 \mathrm{~nm}$ control beam were generated by two laser sources. We chose this wavelength combination because of the much larger absorption of Bio-Te at
$980 \mathrm{~nm}$ than at $1.55 \mu \mathrm{m}$ as shown in Fig. 1f. After the laser sources, two fiber polarization controllers (PCs) were used to adjust the polarization of the signal beam and control beam independently. Both beams were combined using a $0.98 /$ $1.55 \mu \mathrm{m}$ wavelength de-multiplexer (WDM) and were channeled into the Bio-Te-PMMA coated fiber connectors. Owing to the thermo-optical effect, Bio-Te introduced polarizationdependent phase shifts in the two orthogonal components of the $1.55 \mu \mathrm{m}$ signal beam. These two components interfered with each other when they reached the polarizer. This interference created a variable signal output after the polarizer, which was depended upon the power of the control beam.

Figure $5 \mathrm{~b}$ represents the output of the $1.55 \mu \mathrm{m}$ pulse train as modulated by the control beam with a $20 \%$ duty cycle. Compared with a previous report on the use of $\mathrm{WS}_{2}$ as an optical switch ${ }^{39}$, the Bio-Te optical switch presented a significant advantage in the on/off stability mode. Figure $5 \mathrm{c}$ shows the response of the Bio-Te 
optical switch, where the rise and fall times were determined to be $276.3 \mu$ s and $563.0 \mu \mathrm{s}$, respectively, following the $10-90 \%$ rule. These response times suggested almost an order-of-magnitude improvement compared to the values reported in similar devices based on other materials: $\mathrm{WS}_{2}$ (rise time of $7.3 \mathrm{~ms}$ ) as well as graphene optical switches (rise/fall times to be $9.1 \mathrm{~ms} / 3.2 \mathrm{~ms}$ ) ${ }^{39,40}$. The extinction ratio of the Bio-Te optical switch in Fig. $5 \mathrm{c}$ was measured to be $\sim 13 \mathrm{~dB}$. To determine the role of the control pulses, we measured the output waveform of the Bio-Te optical switch as a function of the energy/power of the control pulses. As shown in Fig. 5d, e, the switching time of the signal light depended on the peak power of the pump light but was virtually negligible with the duration time of the control light. These phenomena were consistent with previous experimental measurements reported on other thermo-optic devices ${ }^{39}$, implying that the thermo-optic effect was the dominant mechanism in our BioTe optical switch.

To obtain the thermo-optic coefficient, we performed a series of temperature-dependent ellipsometry measurements on the Bio-Te-PMMA thin film on a silicon substrate. The results are shown in Fig. 5f, and the refractive indexes at $1.55 \mu \mathrm{m}$ as a function of temperature are summarized in the inset. The corresponding thermo-optic coefficient of the Bio-Te-PMMA film is fit to be approximately $-1.64 \times 10^{-4} \mathrm{~K}^{-1}$ by linear regression. The thermo-optic coefficient of the Bio-Te nanocrystal was then estimated to be approximately $-3.5 \times 10^{-3} \mathrm{~K}^{-1}$ (see Supplementary Note 3 ). The previously reported thermos-optic coefficients of graphene and $\mathrm{WS}_{2}$ are $\sim 1.1 \times 10^{-5} \mathrm{~K}^{-1}$ and $\sim 3.4 \times$ $10^{-4} \mathrm{~K}^{-1}$, respectively ${ }^{39,40}$. The magnitude of the thermo-optic coefficient of Bio-Te is 1-2 orders larger than that of graphene and $\mathrm{WS}_{2}$, implying that Bio-Te can introduce much larger phase changes through the thermo-optic effect than graphene or $\mathrm{WS}_{2}$ with the same thickness.

To experimentally determine which of the materials mentioned above could provide the faster performance, we fabricated various PMMA thin films incorporated with Bio-Te, Chem-Te, graphene, and $\mathrm{WS}_{2}$ flakes, and performed comparative studies on the responses of the thermo-optic switches based on the material used. Figure $5 \mathrm{~g}$ shows their output waveforms with $20 \%$ duty cycle. The extinction ratios were measured to be $13.0 \mathrm{~dB}, 12.8 \mathrm{~dB}$, $10.6 \mathrm{~dB}$, and $7.5 \mathrm{~dB}$ for Bio-Te, Chem-Te, graphene and $\mathrm{WS}_{2}$, respectively. We also studied the response speeds of the thermooptic switches using different materials. Figure $5 \mathrm{f}$ shows the enlarged falling edges of the waveforms. We focused on the falling edges here because they are less steep than the rising edges and the slower processes are more likely to create bottlenecks in highspeed response applications. Employing the power law, $y \propto t^{-\gamma}$, all the falling edges were well fitted (as shown by the solid lines in Fig. 5h). The exponent, $\gamma$, was determined to be 0.551, 0.574, 0.396, and 0.201 for Bio-Te, Chem-Te, $\mathrm{WS}_{2}$, and graphene, respectively, where the larger exponent indicates faster heat dissipation. We determined the respective fall times of the Bio-Te and Chem-Te samples to be $764 \mu$ s and $476 \mu$ s, while those of the $\mathrm{WS}_{2}$ and graphene samples were $1.440 \mathrm{~ms}$ and $2.200 \mathrm{~ms}$. These results suggested that tellurium has obvious advantages over graphene and $\mathrm{WS}_{2}$ for high-speed thermo-optic switching applications.

We successfully harvested biologically generated elemental tellurium nanocrystals (Bio-Te) produced by the anaerobe, Bacillus selenitireducens, and studied their potential for nonlinear optical applications that includes optical limiting, mode-locking, Q-switching, and all-optical switching. Bio-Te formed a stable conjugated polymer composite with PmPV, enabling us to construct solid-state devices. We observed a strong saturable absorption in the visible, near-, and mid- infrared optical regimes, indicating that Bio-Te is superior to graphene at $800 \mathrm{~nm}, 2.5 \mu \mathrm{m}$, and $2.8 \mu \mathrm{m}$ wavelengths. In addition, Bio-Te-PmPV composites exhibited strong nonlinear extinction responses to nanosecond pulses, which exceeded those of a $\mathrm{tBu}_{4} \mathrm{PcZn}$ solution, $\mathrm{C}_{60}$ fullerene, single-walled carbon nanotubes, and graphene. By using Bio-Te as a saturable absorber, we successfully generated ultrafast pulse trains by mode-locking/Q-switching in a $1.5 \mu \mathrm{m}$ erbium-doped fiber laser and a $2 \mu \mathrm{m}$ Tm: YAP solid-state lasers, with comparable performance to black phosphorus in broadband and short pulse durations. Finally, we demonstrated a superior all-optical switching phenomenon based on the Bio-Te's thermooptic effects of Bio-Te in optical fiber. This optical switch exhibited excellent performance, with $276.3 \mu$ s and $563.0 \mu$ s rise and fall times, respectively. The thermo-optic coefficient of BioTe was estimated to be approximately $-3.5 \times 10^{-3} \mathrm{~K}^{-1}$, whose magnitude is $1-2$ orders greater than those of graphene and $\mathrm{WS}_{2}$. By comparative studies in thermal-optic switching, we proved that Bio-Te provides obvious improvements in the thermal-optic decaying lifetime compared to $\mathrm{WS}_{2}$ and graphene. Our results imply that Bio-Te is a promising bio-material for a broad range of photonic applications.

\section{Methods}

Synthesis of biological tellurium. Biological tellurium (Bio-Te) was synthesized by growing haloalkaliphilic anaerobic bacterium, Bacillus Selenitireducens, in a lactate-tellurite medium ${ }^{9,10}$. Briefly recounted, growth of B. Selenitireducens was conducted by pulsed additions of $0.6 \mathrm{mM}$ of Te(IV). The culture was incubated statically at $28^{\circ} \mathrm{C}$ for 30 days. During growth $\mathrm{Te}(0)$ nano-rods first appeared on the cell surfaces, and then aggregated into larger $\mathrm{Te}(0)$ shards. These shards sloughed from the cell and finally accumulated as Te rosettes. To separate the $\mathrm{Te}(0)$ rosettes from the bacteria, a lysozyme treatment combined with ultrasonication was employed to break open the bacterial cells. After repeated washing and centrifugation, we obtained assemblages of $\mathrm{Te}(0)$ rosettes which were free of cellular debris (Bio-Te). The purified $\mathrm{Bio}-\mathrm{Te}(0)$ rosettes were stored under a nitrogen atmosphere to preclude oxidation for further experiments.

Fabrication of Bio-Te-PMMA films. $1 \mathrm{~mL}$ of $4 \%$ PMMA-toluene solution was drop-cast into polymer petri dishes ( $55 \mathrm{~mm}$ diameter). After the evaporation of the solvent, PMMA film was formed. Next, another $1 \mathrm{~mL}$ of Bio-Te-PmPV solution was drop-cast onto the surface of the PMMA film. After drying at room temperature for $\sim 10 \mathrm{~min}$, the composite film of Bio-Te/PMMA film was obtained.

Characterization. The crystal structure of the synthesized Bio-Te was studied using high-resolution transmission electron microscopy (HRTEM) on a FEI Tecnai G220 instrument operating at $200 \mathrm{kV}$. The sample for TEM study was prepared by dispersing the powder in absolute ethanol followed by drop-casting the solution onto a 400-mesh copper grid. Raman spectroscopy was carried out on a Jobin Yvon LabRam 1B Raman spectrometer with a He: Ne laser operating at $633 \mathrm{~nm}$. Absorption spectra were taken using a PerkinElmer Lambda 750 instrument. PL decay kinetics were measured from a home-built confocal micro-PL spectrometer integrated with an SFM (Catalyst II, Bruker). The samples for PL measurements were fabricated by drop-casting Bio-Te-PmPV in toluene onto cleaned quartz substrates. The thermo-optic coefficients of Bio-Te-PMMA films was measured in a spectroscopic ellipsometer with variable temperature measurement capability (HORIBA Scientific UVISEL 2).

Z-scans. An open-aperture Z-scan system was used to study the ultrafast NLO behavior of the Te/PmPV composite. This system measures the total transmittance through a sample as a function of incident laser intensity, while the sample is sequentially moved through the focus of a lens (along the $\mathrm{z}$-axis). For the visibleNIR Z-scans, a mode-locked Ti:Sapphire laser operating at $800 \mathrm{~nm}$ with $100 \mathrm{fs}$ pulses at a $1 \mathrm{kHz}$ repetition rate, and a mode-locked fiber laser operating at 1030 $\mathrm{nm}$ and $515 \mathrm{~nm}, 340 \mathrm{fs}, 100 \mathrm{~Hz}$ repetition rate were employed. For the mid-IR Zscans we used the idler beam of the Topaz-Prime OPA output pumped by Spitfire Ace laser system $(800 \mathrm{~nm}, 37 \mathrm{fs}, 7 \mathrm{~mJ}$ pulse energy and $1 \mathrm{kHz}$ repetition rate). The laser pulse energy was determined from average power measured by a thermal power sensor (Thorlabs S401C). A PbS photodetectors (Thorlabs PDA30G) were used to register the incident and transmitted beam signals.

Bio-Te EDF laser. The laser cavity was a fiber ring cavity including EDF, 980/1550 WDM, BioTe saturable absorber, polarization controllers, output coupler, and isolator. The EDF (model LIEKKI Er110 8/125) was 0.3-meter length with $110 \mathrm{~dB} /$ $\mathrm{m}$ absorption at $1530 \mathrm{~nm}$, which was pumped at $980 \mathrm{~nm}$ with $720 \mathrm{~mW}$ maximum power. The output coupler provided a $10 \%$ output ratio. The isolator guaranteed the unidirectional operation of the laser. The entire cavity length was $10.6 \mathrm{~m}$. 


\section{Data availability}

All data are available from the corresponding author upon reasonable request.

Received: 8 October 2018 Accepted: 29 July 2019

Published online: 04 September 2019

\section{References}

1. Novoselov, K., Mishchenko, A., Carvalho, A. \& Neto, A. C. 2D materials and van der Waals heterostructures. Science 353, aac9439 (2016).

2. Reed, E. J. computational Materials Science: Two-dimensional tellurium. Nature 552, 1-2 (2017).

3. Zhu, Z. et al. Multivalency-driven formation of te-based monolayer materials: a combined first-principles and experimental study. Phys. Rev. Lett. 119, 106101 (2017).

4. Liu, Y., Wu, W. \& Goddard, W. A. III Tellurium: fast electrical and atomic transport along the weak interaction direction. J. Am. Chem. Soc. 140, 550-553 (2018).

5. Hirayama, M., Okugawa, R., Ishibashi, S., Murakami, S. \& Miyake, T. Weyl node and spin texture in trigonal tellurium and selenium. Phys. Rev. Lett. 114, 206401 (2015).

6. Wu, B., Liu, X., Yin, J. \& Lee, H. Bulk $\beta$-Te to few layered $\beta$-tellurenes: indirect to direct band-Gap transitions showing semiconducting property. Mater. Res. Express 4, 095902 (2017).

7. Qiao, J. et al. Few-layer Tellurium: one-dimensional-like layered elementary semiconductor with striking physical properties. Sci. Bull. 63, 159-168 (2018).

8. Chen, J. et al. Ultrathin $\beta$-tellurium layers grown on highly oriented pyrolytic graphite by molecular-beam epitaxy. Nanoscale 9, 15945-15948 (2017).

9. Blum, J. S., Bindi, A. B., Buzzelli, J., Stolz, J. F. \& Oremland, R. S. Bacillus arsenicoselenatis, sp. nov., and Bacillus selenitireducens, sp. nov.: two haloalkaliphiles from Mono Lake, California that respire oxyanions of selenium and arsenic. Arch. Microbiol. 171, 19-30 (1998).

10. Baesman, S. M. et al. Formation of tellurium nanocrystals during anaerobic growth of bacteria that use Te oxyanions as respiratory electron acceptors. Appl. Environ. Microbiol. 73, 2135-2143 (2007).

11. Oremland, R. S. et al. Structural and spectral features of selenium nanospheres produced by Se-respiring bacteria. Appl. Environ. Microbiol. 70, 52-60 (2004)

12. Presentato, A. et al. Assembly, growth and conductive properties of tellurium nanorods produced by Rhodococcus aetherivorans BCP1. Sci. Rep. 8, 3923 (2018).

13. Baesman, S., Stolz, J., Kulp, T. \& Oremland, R. S. Enrichment and isolation of Bacillus beveridgei sp. nov., a facultative anaerobic haloalkaliphile from Mono Lake, California, that respires oxyanions of tellurium, selenium, and arsenic. Extremophiles 13, 695-705 (2009).

14. Wang, X. et al. Programming cells for dynamic assembly of inorganic nanoobjects with spatiotemporal control. Adv. Mater. 30, 1705968 (2018).

15. Liao, K.-S. et al. Strong nonlinear photonic responses from microbiologically synthesized tellurium nanocomposites. Chem. Phys. Lett. 484, 242-246 (2010).

16. Narayanan, K. B. \& Sakthivel, N. Biological synthesis of metal nanoparticles by microbes. Adv. Colloid Interface Sci. 156, 1-13 (2010).

17. Curran, S. A. et al. A composite from poly (m-phenylenevinylene-co-2, 5dioctoxy-p-phenylenevinylene) and carbon nanotubes: a novel material for molecular optoelectronics. Adv. Mater. 10, 1091-1093 (1998).

18. Reitz, J. R. Electronic band structure of selenium and tellurium. Phys. Rev. 105, 1233 (1957).

19. Song, J.-M. et al. Superlong high-quality tellurium nanotubes: synthesis, characterization, and optical property. Cryst. Growth Des. 8, 1902-1908 (2008).

20. Gautam, U. K. \& Rao, C. Controlled synthesis of crystalline tellurium nanorods, nanowires, nanobelts and related structures by a self-seeding solution process. J. Mater. Chem. 14, 2530-2535 (2004).

21. Caldwell, R. S. \& Fan, H. Optical properties of tellurium and selenium. Phys. Rev. 114, 664 (1959).

22. Lu, S. et al. Broadband nonlinear optical response in multi-layer black phosphorus: an emerging infrared and mid-infrared optical material. Opt. Express 23, 11183-11194 (2015).

23. Hanlon, D. et al. Liquid exfoliation of solvent-stabilized few-layer black phosphorus for applications beyond electronics. Nat. Commun. 6, 8563 (2015).

24. Bao, Q. et al. Atomic-layer graphene as a saturable absorber for ultrafast pulsed lasers. Adv. Funct. Mater. 19, 3077-3083 (2009).
25. Wang, G. et al. Ultrafast Nonlinear Optical Properties of a Graphene Saturable Mirror in the $2 \mu \mathrm{m}$ wavelength region. Laser Photonics Rev. 11, 1700166 (2017).

26. Martinez, A. \& Sun, Z. Nanotube and graphene saturable absorbers for fibre lasers. Nat. Photonics 7, 842-845 (2013).

27. Malouf, A., Henderson-Sapir, O., Set, S., Yamashita, S. \& Ottaway, D. J. Twophoton absorption and saturable absorption of mid-IR in graphene. Appl. Phys. Lett. 114, 091111 (2019).

28. Wang, J., Hernandez, Y., Lotya, M., Coleman, J. N. \& Blau, W. J. Broadband nonlinear optical response of graphene dispersions. Adv. Mater. 21, 2430-2435 (2009).

29. Tutt, L. W. \& Kost, A. Optical limiting performance of C60 and C70 solutions. Nature 356, 225-226 (1992).

30. Chen, P. et al. Electronic structure and optical limiting behavior of carbon nanotubes. Phys. Rev. Lett. 82, 2548 (1999).

31. Loh, K. P., Bao, Q., Eda, G. \& Chhowalla, M. Graphene oxide as a chemically tunable platform for optical applications. Nat. Chem. 2, 1015-1024 (2010).

32. Zhu, J. et al. Graphene oxide covalently functionalized with zinc phthalocyanine for broadband optical limiting. Carbon 49, 1900-1905 (2011).

33. Sun, Z. et al. Graphene mode-locked ultrafast laser. ACS Nano 4, 803-810 (2010).

34. Wu, K., Zhang, X., Wang, J., Li, X. \& Chen, J. W. S. 2 as a saturable absorber for ultrafast photonic applications of mode-locked and Q-switched lasers. Opt. Express 23, 11453-11461 (2015).

35. Chen, Y. et al. Mechanically exfoliated black phosphorus as a new saturable absorber for both Q-switching and mode-locking laser operation. Opt. Express 23, 12823-12833 (2015).

36. Wang, K. et al. Ultrafast nonlinear excitation dynamics of black phosphorus nanosheets from visible to mid-infrared. ACS Nano 10, 6923-6932 (2016).

37. Willner, A. E., Khaleghi, S., Chitgarha, M. R. \& Yilmaz, O. F. All-optical signal processing. J. Light. Technol. 32, 660-680 (2014).

38. Almeida, V. R., Barrios, C. A., Panepucci, R. R. \& Lipson, M. All-optical control of light on a silicon chip. Nature 431, 1081-1084 (2004).

39. Wu, K. et al. All-optical phase shifter and switch near 1550nm using tungsten disulfide (WS2) deposited tapered fiber. Opt. Express 25, 17639-17649 (2017).

40. Gan, X. et al. Graphene-assisted all-fiber phase shifter and switching. Optica 2, 468-471 (2015)

\section{Acknowledgements}

K.P.W. is supported in part at the Technion by a fellowship from the Lady Davis Foundation. J.W. thanks the financial support received from the National Natural Science Foundation of China (NSFC, 61675217, 61875213, 11874370), the Strategic Priority Research Program of CAS (XDB16030700), the Key Research Program of Frontier Science, CAS (QYZDB-SSW-JSC041), and the Program of Shanghai Academic Research Leader (17XD1403900). W.J.B. gratefully acknowledges a visiting professorship for international scientists from the Chinese Academy of Sciences. His contribution towards this publication has also emanated from research supported in part by a research grant from Science Foundation Ireland (SFI) under Grant Number 12/IA/1306. S.M.B. and R.S.O. were supported by the National Research Program of the Water Mission Area of the U.S. Geological Survey. I.M.K. is supported by President's International Fellowship Initiative (PIFI) of CAS (2017VTB0006, 2018VTB0007). Mention of brand-name products does not constitute an endorsement by the USGS. Curran, Liao, and Maharjan would like to acknowledge the assistant from Integricote in funding materials and providing knowhow. The help from Dr. Guohang Hu on ellipsometry measurements is greatly appreciated.

\section{Author contributions}

J.W., W.J.B., and S.A.C. conceive the original idea. J.W., K.P.W. and L.Z. led the project and wrote the paper. S.M.B., K.L., S.M., S.A.C. and R.S.O. cultivated the bacteria and prepared all the nanocrystals. K.P.W., J.W., I.M.K., Q.Z. and G.W. performed the nonlinear optical measurements. J.W., X.Y.Z., and J.F. prepared the dispersions, Te-PMMA films and measured the thermo-optical coefficients. N.D. S.F.Z. and J.F. performed the spectroscopy and the time-resolved PL. H.Z., J.W. and X.Y.Z. preformed the HR-TEM. X.Z., J.D., and Y.L. built the solid-state laser and measured the performance of Q-switching and mode-locking. K.W. and J.C. measured the performance of Bio-Te Q-switched/mode-locked fiber laser and performed thermo-optical switching measurements. K.P.W., J.W., I.M.K. and K.W. contributed the analysis of the data. All authors discussed the results and commented on the paper. 


\section{Additional information}

Supplementary Information accompanies this paper at https://doi.org/10.1038/s41467019-11898-z.

Competing interests: The authors declare no competing interests.

Reprints and permission information is available online at http://npg.nature.com/ reprintsandpermissions/

Peer review information: Nature Communications would like to thank Wei Ji and other, anonymous reviewers for their contribution to the peer review of this work. Peer review reports are available.

Publisher's note: Springer Nature remains neutral with regard to jurisdictional claims in published maps and institutional affiliations. (c) (i) Open Access This article is licensed under a Creative Commons Attribution 4.0 International License, which permits use, sharing, adaptation, distribution and reproduction in any medium or format, as long as you give appropriate credit to the original author(s) and the source, provide a link to the Creative Commons license, and indicate if changes were made. The images or other third party material in this article are included in the article's Creative Commons license, unless indicated otherwise in a credit line to the material. If material is not included in the article's Creative Commons license and your intended use is not permitted by statutory regulation or exceeds the permitted use, you will need to obtain permission directly from the copyright holder. To view a copy of this license, visit http://creativecommons.org/ licenses/by/4.0/.

(C) The Author(s) 2019 